\title{
Genetically closely related azole-resistant Candida tropicalis in environments can be a threat to healthcare
}

Zi-Li Zhou, MSc $;{ }^{1}$ Jyh-Nong Tsai, PhD; ${ }^{3}$ Min-Nan Tseng, PhD $;{ }^{4}$ Hsing-Lung Liu, MSc $;{ }^{5}$ Chih-Chao Lin, MSc $;{ }^{6}$ Kai-Ting Chen, MSc; ${ }^{6}$ Kuan-Chung Huang, MSc; ${ }^{6}$ Chun-Hua Huang MSc; ${ }^{6}$ Wen-Li Chu, MSc $;{ }^{6}$ Yin-Zhi Chen, MSc; ${ }^{6}$ Feng-Chi Chen, PhD; ${ }^{7}$ Ming-Kung Hsu, MSc; ${ }^{7}$ Si-Chong Wang, Mr; ;,8 Hwei-Ling Peng, PhD; ${ }^{1,2}$ Yun-Liang Yang, PhD; ; ${ }^{12}$ Yee-Chun Chen, M.D., PhD; ${ }^{6,9}$ Hsiu-Jung Lo, PhD; ;,10

\section{Abstract}

\section{Background}

Genetically closely related DST types of human pathogen Candida tropicalis strains with reduced drug susceptibility in humans, fruits, and soils were detected previously. We would like to investigate the diversity of yeasts recovered from farms and the farmers. Furthermore, we characterized the genetic relatedness of azole-resistant $C$. tropicalis recovered from environments and patients.

Methods

Yeasts recovered from farms (fruits, soils, and water) and the farmers (armpit swabs, hand swabs, and oral rise) were identified by ribosomal DNA sequencing and the drug susceptibilities of $C$. tropicalis were determined by broth microdilution assay. Multilocus sequence typing was performed to explore the genetic relatedness of $C$. tropicalis recovered from agricultural and clinical settings.

Results

Of 691 yeast isolates, comprised of 25 genera from 81 farms, 444 (64.3\%) were recovered from environments. Among 85 species, 28 (32.9\%) species including 286 isolates, have been reported to cause diseases in humans. Of the 5 Candida species commonly causing diseases in humans, $C$. krusei $(26 / 30,86.7 \%, p=0.011)$ and $C$. tropicalis $(59 / 65,90.8 \%, p=0.000003)$ were prevalent in environments, whereas $C$. albicans $(37 / 39,94.9 \%, p<0.0000001)$, C. glabrata $(3 / 3,100 \%, p=0.046)$, and C. parapsilosis $(29 / 30,96.7 \%, p<0.0000001)$ were in humans. Interestingly, 9 of the 10 fluconazoleresistant and 20 of the 23 triadimenol-resistant $C$. tropicalis were belonged to diploid sequence type (DST) clade A. Importantly, 22 of the 22 fluconazole-resistant and 38 of the 40 triadimenol-resistant clinical C. tropicalis collected in Taiwan Surveillance of Antimicrobial Resistance of Yeasts (TSARY) in 2014 belonged to clade A.

Conclusion

We have recovered pathogenic yeasts commonly causing diseases in humans from environments. The fact that genetically closely related fluconazole-resistant $C$. tropicalis exists in both environments and patients emphasizes a potential route for pathogenic yeasts to be transmitted from environments to humans and vice versa.

Table 1. FARM 2012: distribution of 691 yeast isolates from environments and farmers

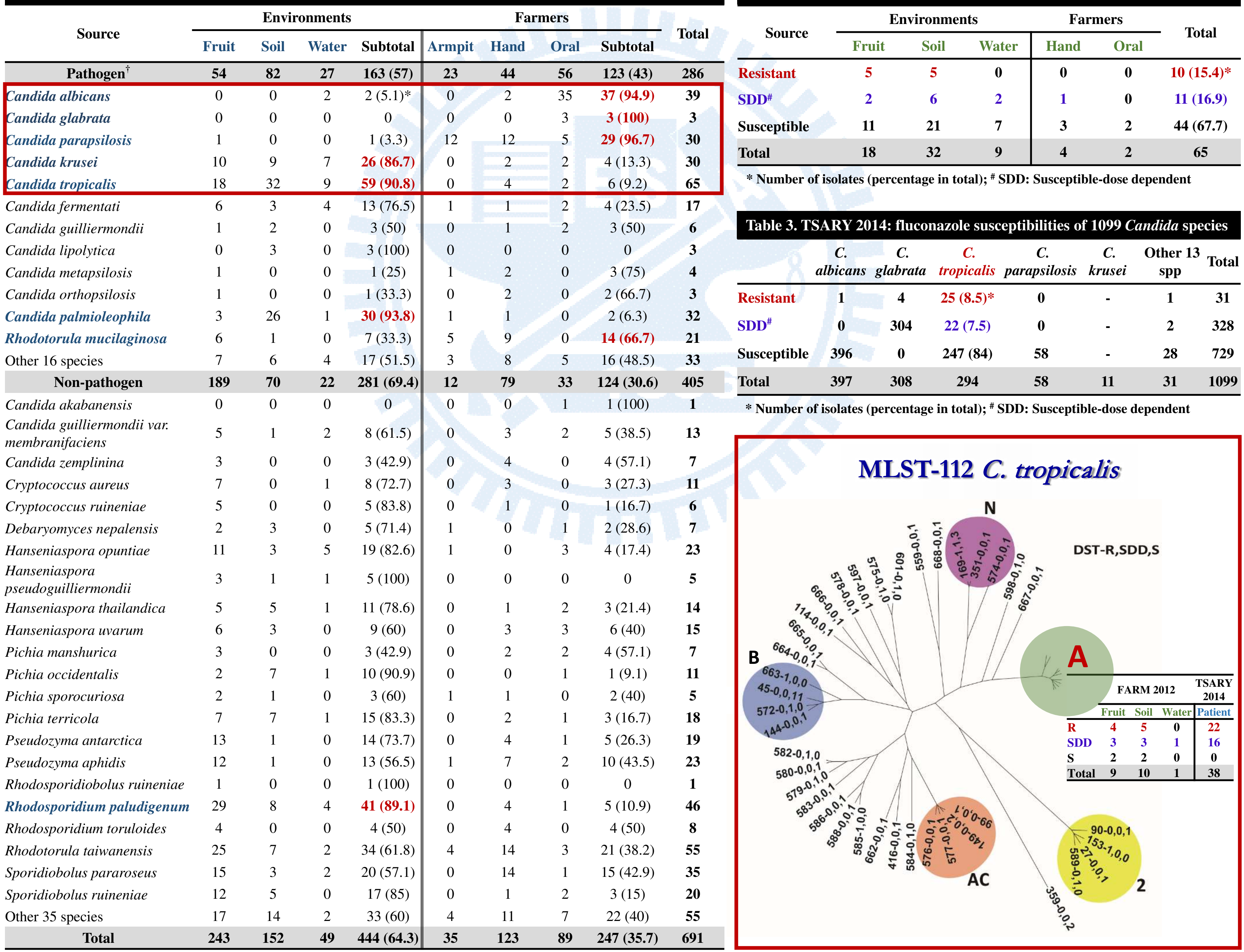

thas been reported to cause diseases in humans. $\quad *$ Number of isolates (percentage in the same species).

\section{Summary}

$>$ Candida tropicalis was the most prevalent non-albicans Candida species in Taiwan and had a high fluconazole resistant rate.

$>9$ of the 10 fluconazole resistant $C$. tropicalis collected in FARM belonged to clade A. Importantly, all the fluconazole resistant $C$. tropicalis collected from patients in TSARY 2014 also belonged to clade A.

$>$ The genetically closely related fluconazole resistant $C$. tropicalis can be transmitted between environments and humans.

$>$ The use of azole drugs in both medical treatment and agriculture needs more attention. 